\title{
Tratamiento de la púrpura trombótica trombocitopénica mediante aféresis terapeútica en paciente con lupus eritematoso sistémico
}

\author{
Jesús Márquez Benítez, Anselmo Guareño Antúnez, Cándida Santos Ugía, Francisca Ramos Jerez, Francisco \\ Calderón Lozano, Jesús Del Arco Adame, Yolanda Marín Pérez, Concepción Cruz Cánovas
}

Enfermeros de la Unidad de Diálisis del Hospital Infanta Cristina de Badajoz

\section{Introducción}

La púrpura trombótica trombocitopénica (PTT) es una entidad englobada en el concepto de microangiopatía trombótica ${ }^{1}$ consistente en la aparición de trombos en la microcirculación que provocan hemólisis, dando lugar a un descenso de hematíes y plaquetas.

Las causas que provocan la PTT son múltiples², encontrándose entre ellas enfermedades autoinmunes como el Lupus Eritematoso Sistémico (LES) aunque también se han descritos casos hereditarios dentro de la misma familia ${ }^{3}$.

En los niños puede aparecer un proceso infeccioso dos o tres semanas antes de desencadenar el proceso de PTT.

La causa del lupus ( LES ) ${ }^{4}$ es desconocida y su incidencia es diez veces más frecuente en mujeres que en hombres, pero englobada en una enfermedad autoinmune influenciada por factores de genética 0 ambiental, dando síntomas como inflamación de articulaciones, riñones, piel, etc.

El tratamiento de la PTT y LES consiste en la resolución de la causa precipitante (corticoides e inmunosupresores) asociado a tratamiento con plasmaféresis.

\section{Caso clínico}

Mujer de 33 años, que llega a urgencias con un cuadro de astenia, antecedentes de alergias no conocidas, lupus

\footnotetext{
Correspondencia:

Anselmo Guareño Antúnez

C/. Julio Cienfuegos Linares

$N^{\circ} 11$, Bloque 1, portal $2,2^{\circ} \mathrm{A}$

06006. Badajoz

E-mail:yoniantunez@yahoo.es
}

cutáneo con eritema malar sin tratamiento desde hace 8 años y en seguimiento por reumatología. Tiroiditis de Hashimoto. Apendicectomía. Exfumadora desde hace 7 años. No bebedora. No hábitos tóxicos, excepto en tomas de bebidas con quinina (tónicas). Episodios de anemia tras los 2 partos. Cesárea y ligadura de trompas en el $3^{\circ}$.

\section{Enfermedad actual:}

Consulta por intensa astenia y presíncopes (de una semana de evolución), que dificultan la deambulación, junto con anorexia sin pérdida de peso. Menstruación muy duradera (8 días) y abundante. No heces melénicas, palidez cutánea y mucosa. Epistaxis y gingivorragia.

La analítica destaca anemia con $6.7 \mathrm{gr} / \mathrm{dl}$ de hemoglobina, trombopenia con plaquetas de $6.000 / \mathrm{mm}^{3}$. LDH 1696 UI/I. Frotis sanguíneo con presencia de esquistocitos. Resto de exploraciones normales.

Diagnóstico: Según los datos clínicos y de laboratorio, la paciente presenta un cuadro compatible de PTT en el contexto del LES.

A su ingreso se transfunde un concentrado de hematíes y otro de plaquetas. Una hora después de pasar las plaquetas, la paciente comienza con un cuadro de afasia global y desviación de la comisura bucal a la izquierda sin otra focalidad neurológica. Posteriormente habla con lenguaje normal sin focalidad evidente.

Dado el añadido síntoma neurológico se ingresa en hematología para su estudio.

Al presentar la paciente microangiopatía trombótica y crisis convulsivas, nos solicitan desde ese servicio la realización de plasmaféresis. Debido al empeoramiento clínico de la paciente, ingresa en UCI.

Plan de sesiones de plasmaféresis:

El volumen de recambio debe situarse entre $1-1,5$ veces el volumen plasmático.

El tratamiento se prolongará hasta 48 h. después de conseguir la respuesta. 
La paciente pesa $75 \mathrm{kgr}$., y por tablas de relación, le corresponden un total de $3.500 \mathrm{cc}$. de volumen a reinfundir, por lo que se programan: $1.750 \mathrm{cc}$. de plasma fresco +350 cc. de albúmina humana al $20 \%+1.400$ cc. solución Dianeal ${ }^{\circledR} 1,36 \%$ de diálisis peritoneal.

\section{Incidencia de las sesiones:}

Para la realización de la $1^{\text {a }}$ plasmaféresis en UCI se intenta implantar un catéter en yugular derecha, pero debido a su dificultad y exceso de sangrado, se coloca un catéter doble luz en femoral izquierda.

El monitor utilizado para la técnica fue un PRISMA CRRT-TPE (Hospal $\left.{ }^{\circledR}\right)$, con filtro PTE-2000.

La dosis de heparina fue de 15-5-5-5 mgr, siendo la duración de las sesiones de unos 210 minutos, con una infusión media de $980 \mathrm{ml} / \mathrm{h}$, unos flujos de sangre entre 120-140 ml/min .y unas presiones de ( PA. -85 a -170 $\mathrm{mmHg}$, y PV de 70 a $90 \mathrm{mmHg}$, manteniéndose una PTM entre 25-55 $\mathrm{mmHg}$ ).

La paciente en UCI presenta un mal estado general (sedada), con las constantes mantenidas. Se le realizaron un total de 17 sesiones.

\section{Incidencia de las mismas:}

La $1^{a}$ sesión la pasó muy agitada.

En la $5^{\mathrm{a}}$ y $6^{\mathrm{a}}$ sufre hipotensión y convulsiones importantes, que ceden con diazepam y salino fisiológico, terminando el tratamiento.

En la $16^{a}$ hay un aumento de PTM hasta $55 \mathrm{~mm} \mathrm{Hg}$ faltando 50 minutos, finalizando la sesión.

En la $17^{\text {a }}$, hay un fallo del test de aire cuando faltan 90 minutos, por lo que hubo que cambiar el SET.

El resto de las sesiones se realizaron sin problemas, siendo bien toleradas por la paciente.

\section{Evolución}

En cuanto a la evolución de la $\mathrm{Hb}$ y plaquetas, así como LDH, se detallan en el cuadro adjunto.

La LDH nos da el grado de hemólisis. A más hemólisis mayor LDH.

La evolución de las Hb. Plaquetas y LDH mejoraron con las sesiones de plasmaféresis, pero en la $1 l^{\text {a }}$ sesión se decide aparte de seguir con la plasmaféresis, poner un tratamiento coadjuvante con RITUXIMAB Y MICOFENOLATO, subiendo las plaquetas a $>150.000 / \mathrm{mm} 3$, quedando suspendidas las plasmaféresis hasta completar 17 sesiones., debido a la mejora clínica y analítica de la paciente y su posterior alta médica a los 4 días de la última sesión, no presentando ninguna recidiva hasta la fecha. Ver la evolución analítica desde el comienzo con plasmaféresis hasta su alta médica. (tabla 1).

\section{Discusión}

Describimos un caso de una paciente con antecedentes de enfermedad autoinmune como lo demuestra la existencia de Lupus Eritematoso5 más Tiroiditis de Hashimoto que desarrolla un cuadro de anemia, plaquetopenia y elevación de LDH, junto con esquistocitos en frotis de sangre periférica, con diagnóstico de microangiopatía trombótica.

El desarrollo de una microangiopatía trombótica en este contexto se produce como consecuencia de anticuerpos circulantes contra el ADAMS 13, enzima necesaria para el correcto funcionamiento de la coagulación. En nuestra paciente, dado el rápido deterioro clínico y la alta sospecha de PTT en el contexto del Lupus, se inició tratamiento con plasmaféresis inmediatamente sin posibilidad de cuantificar la actividad ADAMS 13.

El tratamiento de la PTT en este contexto va dirigido al tratamiento de la enfermedad de base, en este caso, eliminar los anticuerpos circulantes mediante plasmaféresis e impedir la síntesis de nuevos anticuerpos patológicos mediante tratamiento inmunosupresor (corticoides y micofenolato inicialmente y posteriormente Rituximab dada la escasa mejoría).

A pesar de los importantes avances en el tratamiento médico de la PTT y LES ${ }^{6}$ para evitar la síntesis de anticuerpos, aún es necesario la utilización de la plasmaféresis, no sólo para la eliminación de los anticuerpos circulantes iniciales hasta el inicio del efecto farmacológico, sino también para el aporte de ADAMS 13 con la infusión del plasma posterior.

La plasmaféresis es un método de filtración plasmática utilizada para la eliminación de sustancias del plasma de gran peso molecular como anticuerpos, inmunocomplejos inmunes, componentes del complemento ${ }^{7}$, etc. Su utilización en el manejo de enfermedades renales de origen inmunológico se inició a finales del siglo pasado donde se describe por primera vez la mejoría tras la realización de plasmaféresis a un paciente con enfermedad por anticuerpos anti-membrana basal glomerular, ampliándose su utilización a otras enfermedades de base autoinmune como la PTT con el paso de los años.

El recambio plasmático (R.P) debe iniciarse lo antes posible en las primeras $24 \mathrm{~h}$. tras su diagnóstico, pues la demora tiene peor pronóstico.

Los recambios plasmáticos deben realizarse diariamente hasta la normalización de las plaquetas, hemoglobina y la lactatodeshidrogenasa (LDH) en plasma y la desaparición de los síntomas. 
La plasmaféresis puede considerarse como un método seguro ${ }^{8}$. En un estudio con 370 sesiones de plasmaféresis realizadas en 54 pacientes, el principal efecto adverso observado fue la hipotensión en un $8.4 \%$ de las sesiones. En nuestra paciente ocurrió en 2 sesiones sin necesidad de drogas vasoactivas y recuperando normotensión arterial con infusión de salino fisiológico. La aparición de efectos adversos graves ocurrió en el $0.9 \%$ de los casos según el registro Sueco con más de 20.000 procedimientos, con una incidencia de efectos adversos global de tan solo el $4.3 \%$.

\section{Conclusiones}

A pesar de los importantes avances del tratamiento médico en las enfermedades autoinmunes, la plasmaféresis continúa siendo un método imprescindible en los casos graves, considerándose una técnica relativamente segura.

Dada la complejidad de la técnica para el tratamiento de este tipo de patologías, enfermería juega un papel fundamental, siendo de suma importancia que el profesional que la realice disponga de suficiente formación y experiencia, para saber detectar y resolver los síntomas adversos en el paciente y los problemas técnicos que se vayan presentando (inadecuados flujos de sangre, de PA, PV y PTM), con el fin de llevar a término cada recambio plasmático (R.P.), pues de la eficacia de las mismas depende la evolución de la paciente.

\section{Agradecimientos}

A todo el equipo de enfermería de la unidad de HD.

A Sergio Barroso Hernández, nefrólogo de la unidad.

\begin{tabular}{|c|}
\hline Recibido: 22 Abril 2014 \\
Revisado: 30 Abril 2014 \\
Modificado: 20 Mayo 2014 \\
Aceptado: 25 Mayo 2014 \\
\hline
\end{tabular}

\section{Bibliografía}

1. William F. Clark. Thrombotic Microangiopathy: Current Knowledge and Outcomes with Plasma Exchange. Seminairs in Dialysis- Vol 25, No 2 (MarchApril) 2012 pp 214-219.

2. De la Rubia J, Contreras E, Del Rio-Garma J. Púrpura trombótica trombocitopénica. Med Clín (Barc) 2011; 136: 534-40.
3. Purpura trombocitopénico idiopático. Madero L. Molina j. Sevilla J. Controversias, BSCP can ped 2001; 25(2); 291-302 Links.

4. Hahn BH. Tsao BP. Pathogenesis of systemic lupus erithematosus. In; Firestein Budd RC, Harris ED jr, et al, eds. Kelley's Tevtbooi of rheumatology. 8 th. Ed. PhilSWLPHI, Pa; saunders Elsevier; 2008: chap 74.

5. Hahn BH. Systemic lupus erythematosus. In: longo DL, et al. Harrison's on line. 18 th ed. New York: the Mc Graw- Hill companies; 2012.

6. Haija $A j$, et al. The role and effect of complementary and alternative medicine in sistemic lupus eritematosus. Rheumatic Disease clinicsof NBorth América. 2011; 37; 47.

7. Charles D. Pusey. Jeremy B. Levy. Plasmaferésis in immunologia renal disease blood purification 2012; 33; 190-198

8. Szczeklik W et al. Complications in patients treated with plasmapheresis in the intensive care unit. Anaestesiol intensive ther. 2013; 45 (1): 7-13.

Tabla 1. Evolución Analítica

\begin{tabular}{|c|c|c|c|}
\hline Fecha & Hemoglobina $(\mathbf{g} / \mathbf{d})$ & Plaquetas $\left(\mathbf{m i l} / \mathbf{m m}^{3}\right)$ & LDH (UI/I) \\
\hline Día 1 & 6,3 & 25.000 & 1.570 \\
\hline Día 2 & 6,6 & 5.000 & 1.183 \\
\hline Día 3 & 6,4 & 5.200 & 1.205 \\
\hline Día 4 & 6,5 & 6.000 & 1.257 \\
\hline Día 5 & 7,8 & 10.000 & 1.528 \\
\hline Día 6 & 6 & 3.000 & 1.524 \\
\hline Día 7 & 7,4 & 3.000 & 2.072 \\
\hline Día 8 & 7,1 & 3.000 & 1.529 \\
\hline Día 9 & 5,5 & 19.000 & 1.404 \\
\hline Día 10 & 5,2 & 3.000 & 1.340 \\
\hline Día 11 & 7,9 & 9.000 & 1.493 \\
\hline Día 12 & 7,1 & 7.000 & 1.181 \\
\hline Día 13 & 8,3 & 15.000 & 775 \\
\hline Día 14 & 7,7 & 26.000 & 651 \\
\hline Día 15 & 8,5 & 47.000 & 596 \\
\hline Día 16 & 9,3 & 95.000 & 552 \\
\hline Día 17 & 11,3 & 142.000 & 446 \\
\hline Día 18 & 9,3 & 154.000 & 310 \\
\hline Día 19 & 9,2 & 170.000 & 485 \\
\hline Día 20 & 8,9 & 206.000 & 393 \\
\hline Día 21 & 9,5 & 222.000 & 389 \\
\hline & & & \\
\hline
\end{tabular}

